\title{
A spatio-temporal analysis of influenza-like illness in Iran from 2011 to 2016
}

\author{
Mohammad Hossein Panahi ${ }^{1}$, Mahboubeh Parsaeian ${ }^{1}$, Mohammad Ali Mansournia ${ }^{1}$, Mostafa Khoshabi ${ }^{2}$, \\ Mohammad Mehdi Gouya ${ }^{3,4}$, Payman Hemati ${ }^{3}$, Akbar Fotouhi*1(D)
}

Received: 30 Jul 2019

Published: 22 Jun 2020

\section{Abstract}

Background: Investigating the spatial aspects of the disease can help decision-makers and researchers better understand the pattern of the disease, and is also very important in the implementation of the disease control programs. Given the vast area of Iran, as well as the diverse geographical and climate conditions of the country, using the geographical information system (GIS) is a suitable method for the study of influenza. In this study, we provide a clear picture of the distribution of the influenza-like illness (ILI) in Iranian provinces through the years from 2011 to 2016 by applying a spatio-temporal analysis, using geographic information system (GIS). Disease rates by location and year are estimated, and then hot spots and cold spots are distinguished.

Methods: This study was conducted using the ILI incidence rate data recorded in the Iranian Influenza Surveillance System from August 2011 to August 2016. The Choropleth map method and the various equal interval and natural break classifications were used. The local Getis-Ord Gi* method was then used to identify the hot spots and regions where, for some reason, the distribution of the disease had significantly clustered spatially. Statistical analyses were done using the ArcGIS 10.2 software.

Results: This study indicates that the highest ILI rate belongs to the period from August 2014 to August 2015 with a rate of 180.26 (95\%CI: 177.65 to 182.9 ) per 100,000 people. The results show that the highest 5-year mean of ILI rate belongs to Zanjan, Markazi, Lorestan, Ilam, North Khorasan, and South Khorasan provinces. Also, results from the local Getis-Ord Gi* method show that ILI has formed a hot spot between the years 2011 and 2013 on the eastern borders of Iran and afterward during the years 2014 to 2016 in the western regions of the country.

Conclusion: Given the importance of influenza and its huge economic burden on the society, identifying the hot spot regions can help better manage the disease. This study indicates the distribution of the disease has formed a hot spot in the western regions of the country.

Keywords: Epidemiology, Influenza, Surveillance, Spatial analysis, Iran

Conflicts of Interest: None declared

Funding: None declared

\section{*This work has been published under CC BY-NC-SA 1.0 license.}

Copyright $\odot$ Iran University of Medical Sciences

Cite this article as: Panahi MH, Parsaeian M, Mansournia MA, Khoshabi M, Gouya MM, Hemati P, Fotouhi A. A spatio-temporal analysis of influenza-like illness in Iran from 2011 to 2016. Med J Islam Repub Iran. 2020 (22 Jun);34:65. https://doi.org/10.47176/mjiri.34.65

\section{Introduction}

Influenza is an infectious respiratory disease that, due to the likelihood of easy transmission of the disease from one person to another, as well as general or partial antigenic

Corresponding author: Dr Akbar Fotouhi, afotouhi@tums.ac.ir

1. Epidemiology and Biostatistics Department, School of Public Health, Tehran University of Medical Sciences, Tehran, Iran

2. Department of Geo-Spatial Information System (GIS), Center of Excellence in GIS, K.N. Toosi University of Technology, Tehran, Iran

3. Center for Communicable Disease Control, Ministry of Health \& Medical Education, Tehran, Iran

4. Iran University of Medical Sciences, Tehran, Iran changes, can be seen in the forms of pandemics or large or small epidemics (1-3). This disease can easily affect any person in any age group (1) and is annually responsible

$\uparrow$ What is "already known" in this topic:

Influenza can affect any person in any age group, and is annually responsible for the death of 290,000 to 650,000 people in the world. This disease seasonally or once every few years, causes a lot of costs through its pandemics.

\section{$\rightarrow$ What this article adds:}

The results show that the ILI hot spots during the years 2011 to 2013 have been mostly on the eastern borders of Iran, while the hot spots for ILI in recent years have been centered on the western regions of Iran. 
for the death of 290,000 to 650,000 people in the world (1). Since the beginning of the twentieth century, there have been four pandemic influenza cases in the world, the last of which was the 2009 epidemic where the disease was reported in more than 214 countries in the world, imposing, directly and indirectly, huge economic burdens on society (4). Given the importance of influenza, this disease is one of the diseases to be under the surveillance of the World Health Organisation (WHO). In spite of all the measures taken in different countries around the world regarding the monitoring and surveillance of influenza, this disease still, seasonally or once every few years, causes a lot of costs through its pandemics (5-7). Investigating the spatial aspects of the disease can help decision-makers and researchers better understand the pattern of the disease, and is also very important in the implementation of the disease control programs. Given the vast area of Iran, as well as the diverse geographical and climate conditions of the country, using the geographical information system (GIS) is a suitable method for the study of influenza. In this study, we provide a clear picture of the distribution of the influenza-like illness (ILI) in Iranian provinces through the years from 2011 to 2016 by applying a spatiotemporal analysis, using geographic information system (GIS). Disease rates by location and year are estimated, and then hot spots and cold spots are distinguished.

\section{Methods}

\section{Setting and patients}

Iran, with a population of 80 million people, is a country located in West Asia and the WHO Eastern Mediterranean region. Iran's "National Surveillance System for Influenza" was established in 2004. The measures taken in this surveillance system include the recording of acute and severe hospitalized cases of respiratory illnesses and influenza-like illnesses $(8,9)$.

The current Influenza Surveillance System was initiated electronically and at the Infectious Disease Control Center in cooperation with the Ministry of Health's Information Technology Office for the purpose of early reporting, registration of suspected influenza, and post-pandemic care in the country. Registration and data collection and analysis are based on this care system. Diagnosis of patients is made in the same way according to international guidelines and definitions in all universities. In this regard, due to the launch and activation of this system, all patient information is recorded by the universities in the shortest time in the system. The management of the system dashboard for the evaluation of the disease process and the correct recording of the disease at the Ministry of Health's Infectious Disease Management Center is subject to modifications in the ongoing monitoring of the registration system in the event of data conflicts with the university. In this surveillance system, by specifying a sentinel site in each city, cases of influenza-like illnesses are recorded and reported on a weekly basis, the details of which have been published before. This study has been conducted based on the data recorded in the Iranian Influenza Surveillance System (IISS) (8-10). After receiving the data from the Infectious Disease Management Center and be- fore starting the analysis, reviewing and replicating the data based on the national code, name and surname, date of birth and other personal data, as well as the date on which symptoms begin, the city and university reporting patients was performed. Finally, the analysis of this study after data cleaning and the removal of duplicate data was performed on individuals with ILI symptoms registered in the system from August 2011 to August 2016.

\section{Influenza-like illness definition}

Any person with acute respiratory infections with a fever higher than or equal to $38^{\circ} \mathrm{C}$ (oral) and coughing, with the onset of the disease being within the last ten days (11).

\section{Statistical Analyses}

In this study, the ILI rate was calculated based on Formula 1 below:

\section{Formula (1) Incidence rate}

$$
=\frac{\text { The number of cases reported weekly }}{\text { The average population in each year }} \times 100000
$$

Given that the beginning of the influenza season in the northern hemisphere is around October each year and the end of it is in October of the following year, in this study, in order to avoid missing the epidemic seasons, data from August 2011 to August 2016 have been used (12). In this study, ILI rate classification has been done according to the provinces and by using the Choropleth maps, and the two methods of equal intervals and natural break $(13,14)$.

Equal interval: This method divides the data into equal size classes (e.g., 0-10, 10-20, 20-30, etc.), and it works better on data that is spread through the entire range. Equal intervals for classes are commonly considered. Therefore, the difference of maximum value from minimum value was divided by the number of classes to obtain enough length of the interval.

Natural Breaks: This method provides an optimal classification scheme by minimizing within-class variance and maximizing between-class differences to find class breaks. It is based on natural grouping in the nature of data. Fracture points are chosen such that similar disorders are placed in a class as best as possible and enhance the variations between classes (16).

Spatial pattern analysis: In order to identify clusters, the local Getis-Ord Gi* was used as local indicators of spatial autocorrelation (LISA). Clusters are the points where the ILI rate is considerably high $(\mathrm{HH})$ or considerably low (LL) in terms of geo-statistics. Outlier data are regions where the ILI rate is high but are surrounded by regions with low ILI rate (HL), or regions where the ILI rate is low but are surrounded by regions with high ILI rate $(\mathrm{LH})$ (15-17).

In the local Getis-Ord Gi* method, the weight is calculated by taking the neighborhoods into account. Neighbourhood can be considered as adjacency or distance. In addition, factors such as the duration of time needed to travel, or the cost of traveling, can also be defined as 
neighborhood and weight. In this part, the fixed distance band is used to determine the weights, so that the regions which fall outside a fixed and specific distance do not affect the internal regions (15-18). Statistical analyses were done using the ArcGIS 10.2 software.

Hot and cold spot detection: The decision as to whether regard regions as hot or cold spot is made using the GetisOrd Gi* statistic based on $\mathrm{Z}$ score (Formula 2). Regions with a positive $Z$-score and values $\geq 1.96$ indicate a higher rate and are considered as hot spots. Likewise, regions with a negative $Z$-score and values $\leq-1.96$ indicate a lower rate and are cold spots (14-21).

$$
\text { Formula (2) } G_{i}^{*}=\frac{\sum_{j=1}^{n} W_{i, j} x_{j}-\bar{x} \sum_{j=1}^{n} W_{i, j}}{s \sqrt{\frac{\left.n \sum_{j=1}^{n} w_{i, j}^{2}-\left(\sum_{j=1}^{n} w_{i, j}\right)^{2}\right]}{n-1}}}
$$

In this formula, $x_{j}$ is the rate value for the $j$-th area. $W_{i, j}$ is the spatial weight between the $i$-th and $j$-th areas, and $n$ equals the total number of provinces. $\bar{X}$ and $S$ are, respectively, the arithmetic mean and the standard deviation of the ILI rate in $\mathrm{n}$ provinces.

\section{Results}

The ILI rate from week 31 in each year until week 30 of the next year in the period between 2011 to 2016 shows that the lowest and the highest ILI rate belong to the 20132014 and 2014-2015 seasons, respectively, with an ILI rate of 159.11 (95\%CI: 156.66 to 161.60$)$ and 180.26
(95\%CI: 177.65 to 182.9 (per 100,000 people (Fig. 1). It becomes clear that the highest 5-year mean of ILI rate belongs to the provinces of Zanjan, Markazi, Lorestan, Ilam, North Khorasan, and South Khorasan (Fig. 2).

By using the Choropleth map and the two methods of equal interval and natural break, variations in the disease rate were examined. The equal interval method divides the interval between the highest value and the lowest value into equal intervals. In this method, in order to determine the changing trend of the disease in different years, a fixed classification is required. Here, the lowest ILI rate in different years, i.e. zero, was considered as the starting point of the range, and the highest ILI rate in different years, i.e. 128.8 (95\%CI: 126.60 to 131.04 ), as the endpoint of the range. On this basis, the disease rate between the years 2011 through 2016, as well as the means for all years, were classified (Figs. 3 \& 4). The results show that during the years 2011 to 2016, and based on the equal interval method, the ILI rate has increased in some provinces, including Kermanshah and Zanjan. Also, by examining the statistical diagrams and using the equal interval and natural break methods, it becomes clear that the highest 5year mean of ILI rate belongs to the provinces of Zanjan, Markazi, Lorestan, Ilam, North Khorasan and South Khorasan (Fig. 2). Moreover, in this study, in order to identify the disease clusters and hot spots, the local GetisOrd Gi* method was used (Fig. 5). Accordingly, it can be seen that the ILI rate during the seasons 2011 to 2013 in the provinces of Razavi Khorasan and South Khorasan, and during the seasons 2013 to 2016 in the Kermanshah

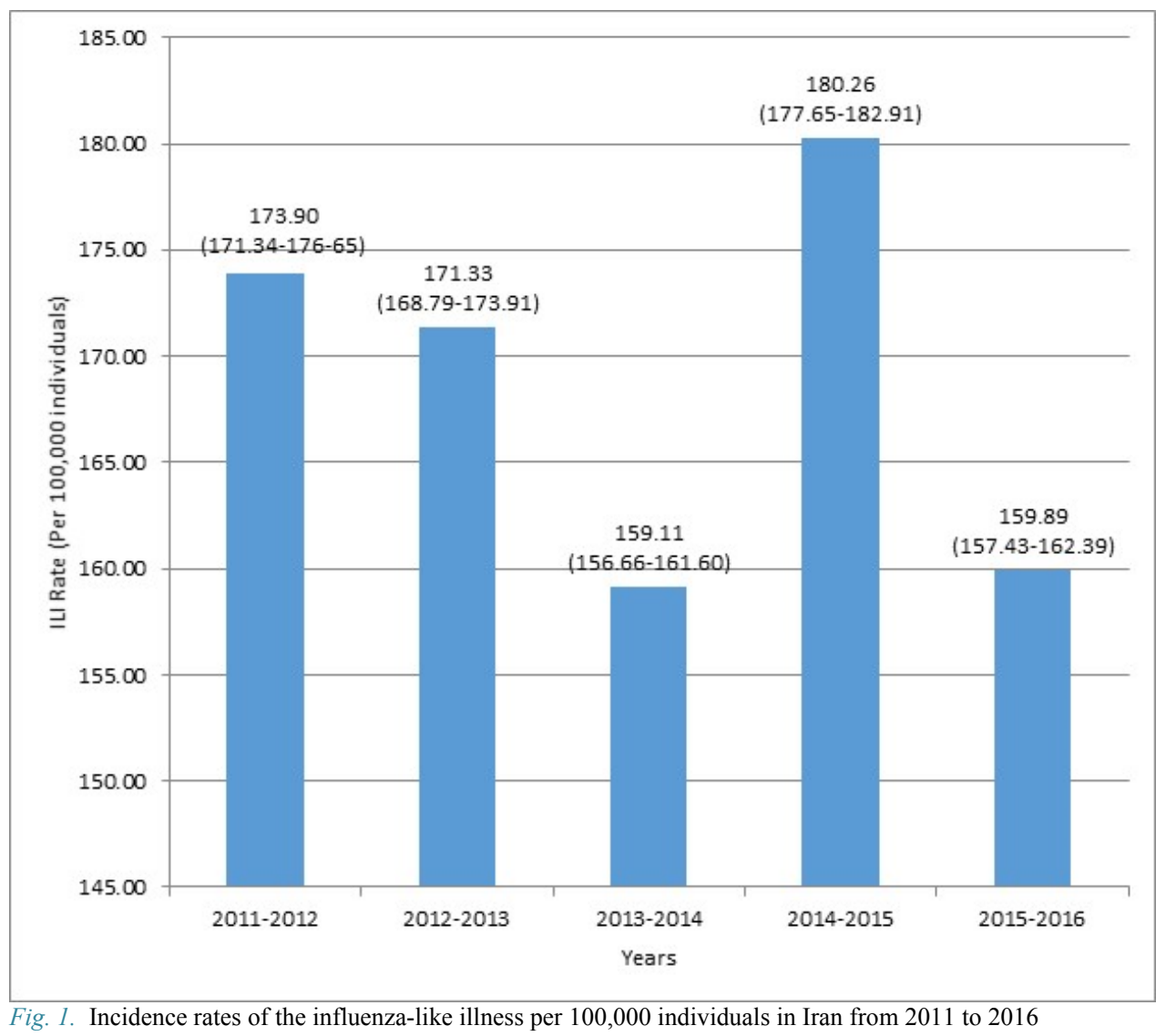




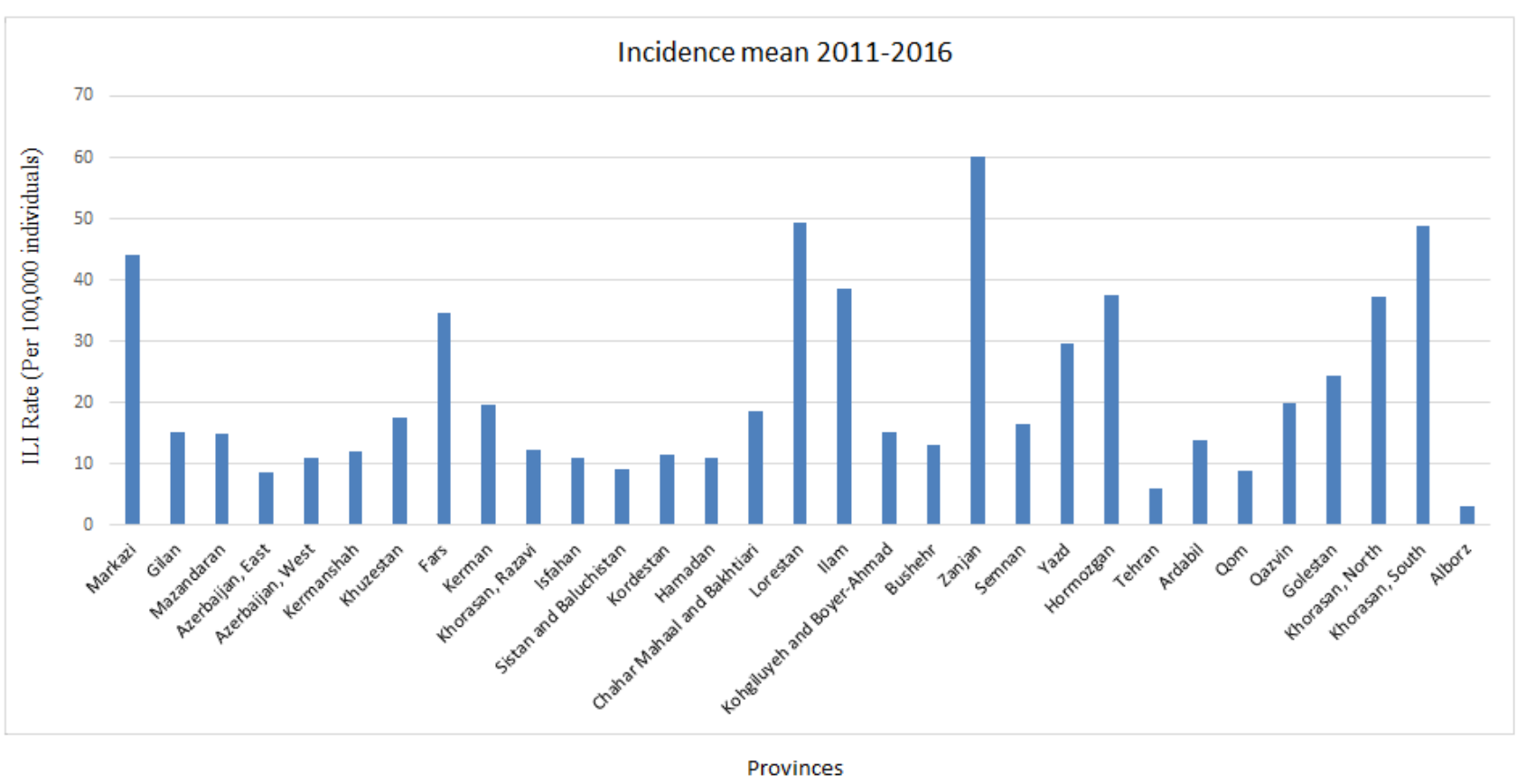

Fig. 2. Mean incidence rates of influenza-like illness per 100,000 individuals in provinces of Iran from 2011 to 2016
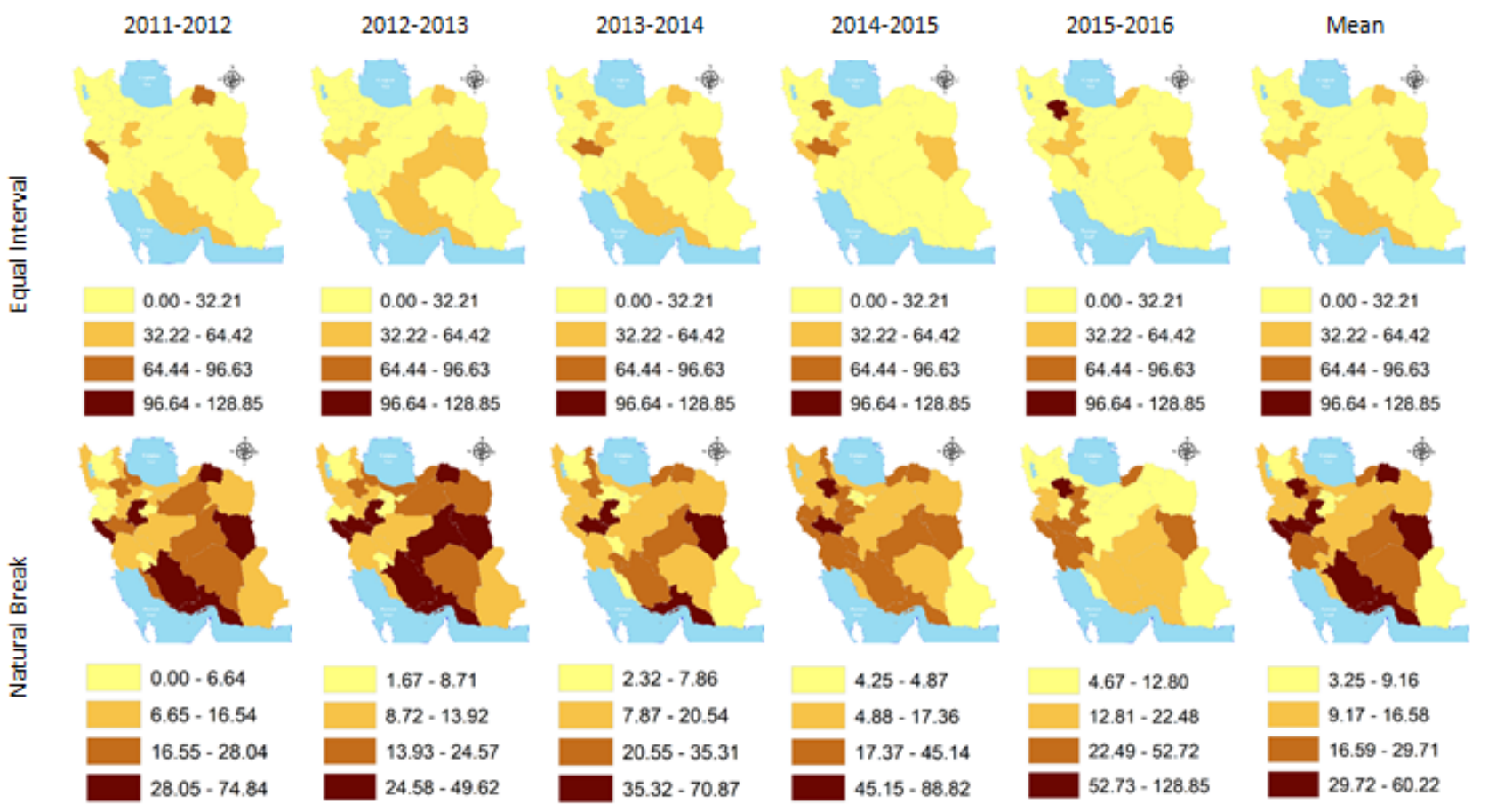

Fig. 3. Choropleth Map of influenza-like illness Incidence Rate by equal interval and natural break clustering approaches from 2011 to 2016: Classes were illustrated by colors.

province, has formed a cluster (a hot spot).

\section{Discussion}

In this study, various analyses in the GIS have been used to understand the distribution of the ILI better. In order to investigate the spatial distribution of ILI changes, the Choropleth map method, together with the classification methods of equal interval and natural break, was used.
Given the scattered status of the ILI in different provinces in Iran and the inclusion of most of the provinces in one class, the equal interval classification method does not yield an appropriate visual interpretation in one year. However, this method makes it possible to observe the growth trend of the disease over different years, due to the fact that the length of the intervals is constant over the years (22). Moreover, the natural break method, with its proper distribution of the number of provinces in each 
Equal Interval

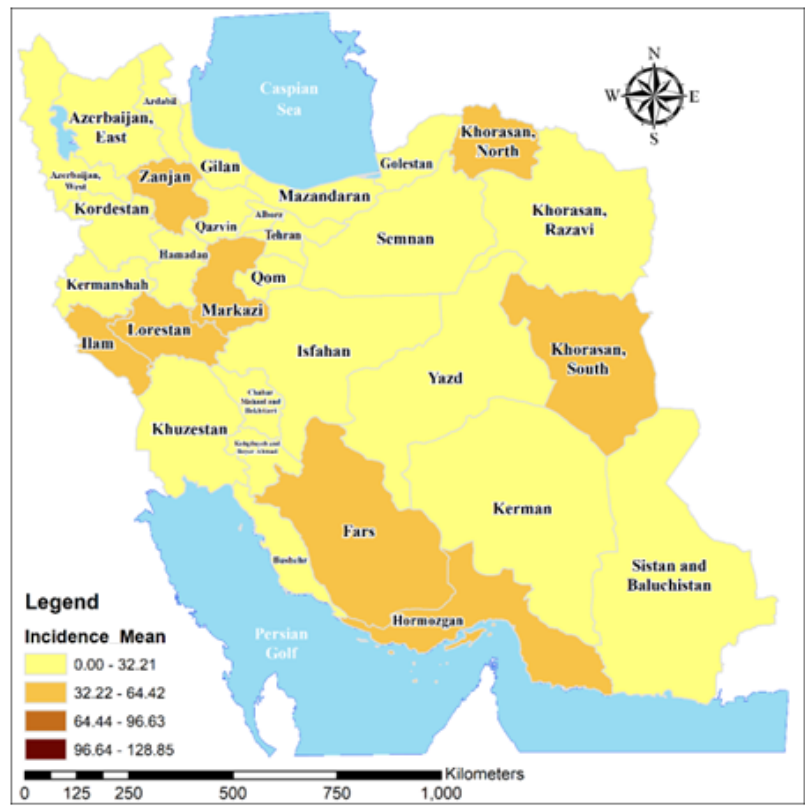

Natural Break

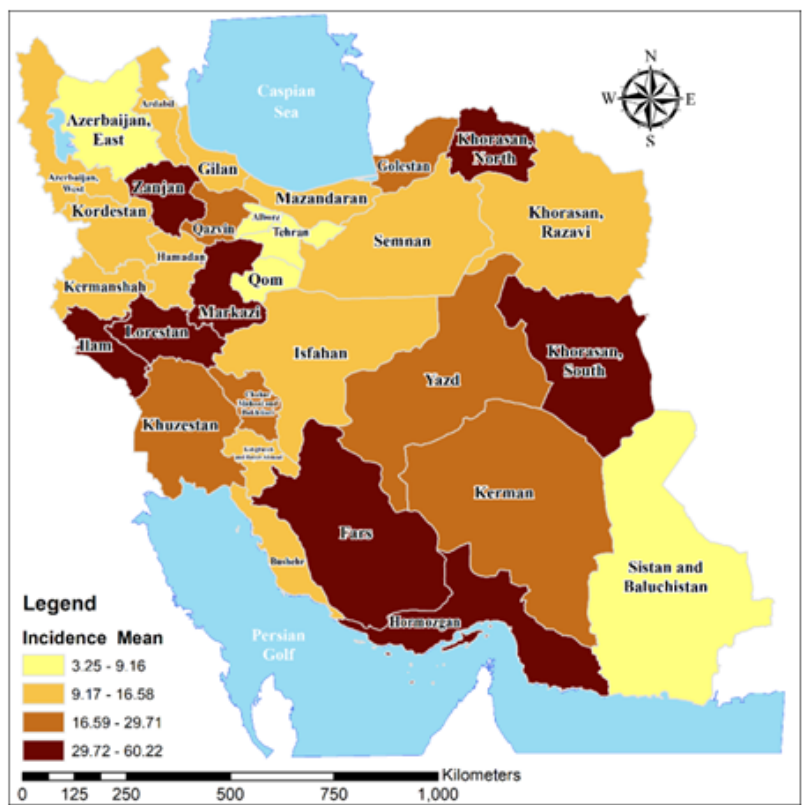

Fig. 4. Choropleth Map by Equal interval and Natural break clustering approaches for incidence mean of influenza-like illness from 2011 to 2016

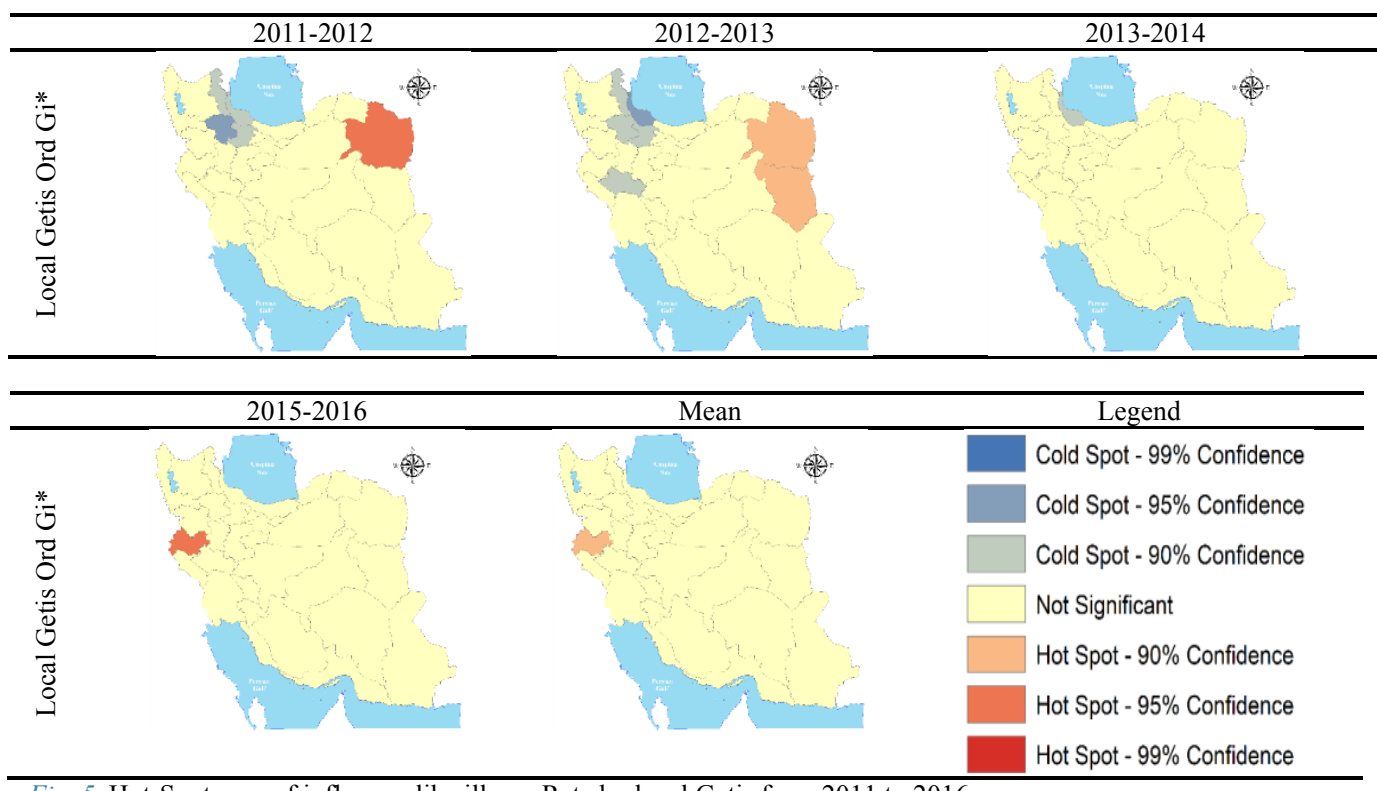

Fig. 5. Hot-Spot map of influenza-like illness Rate by local Getis from 2011 to 2016

class, provides an appropriate visual interpretation of the status of the disease in one year. These results show that the ILI rate has increased during the years 2011 to 2013 in the provinces of Kermanshah and Zanjan. Bearing in mind the vast area of Iran, simply through visual observations, it is not possible to identify the disease clusters, or the hot spots, where examining the disease is of great importance.

Accordingly, in this study, the local Getis-Ord Gi* cluster detection method was used to identify the points where the disease has clustered. The results of this method show that the ILI hot spots during the years 2011 to 2013 have been mostly on the eastern borders of Iran, while from 2014 to 2016 , the clustering of the disease has been in the west of Iran.

Examining the results reveals that the high rate of the disease in a certain province does not necessarily imply that those areas are hot spots, and that the use of appropriate indicators is required to diagnose the clustering of the disease.

Moreover, given the fact that cluster detection analysis is based on points analysis, in the case of entering a region, the center of gravity of that region is generally considered to be representative. Therefore, choosing smaller units can result in more accurate results. Accordingly, given the large size and the heterogeneity of the provinces, it is recommended that in future studies, smaller scales 
be used for analysis in order to identify clusters more accurately. In addition, there is a probability of underestimating the ILI cases in some places in a way that the data were collected, which would be a limitation of this study.

\section{Conclusion}

According to the results of this study, which indicate that the hot spots for influenza in recent years have been centered on the western regions of the country, the disease control authorities can focus on appropriate disease prevention and control measures in these areas. It is hence recommended that the control and research programs for influenza in the western regions of the country be given priority over other regions.

\section{Ethical issues}

This article is part of the Ph.D. thesis at Tehran University of Medical Sciences with ethic code IR.TUMS. SPH.REC.1396.2323.

The study did not involve human participants and/or animals; therefore, no informed consent was needed.

\section{Conflict of Interests}

The authors declare that they have no competing interests.

\section{References}

1. Influenza (Seasonal) [Internet]. Who.int. 2019 [cited 14 May 2019]. Available from: https://www.who.int/en/news-room/fact-sheets/detail/ influenza-(seasonal)

2. Wikramaratna PS, Sandeman M, Recker M, Gupta S. The antigenic evolution of influenza: drift or thrift? Philos Trans R Soc Lond B Biol Sci. 2013;368(1614):20120200.

3. Hampson AW. Influenza virus antigens and 'antigenic drift'. Perspect Med Virol. 2002;7:49-85

4. "WHO | Pandemic (H1N1) 2009 - Update 112". Who.int. N.p., 2016. Web. 10 Nov. 2016.

5. Szucs T. The socio-economic burden of influenza. J Antimicrob Chemother. 1999:44:11-5.

6. Molinari NA, Ortega-Sanchez IR, Messonnier ML, Thompson WW, Wortley PM, Weintraub E, et al. The annual impact of seasonal influenza in the US: measuring disease burden and cost. NPJ Vaccines. 2007;25:5086-5096.

7. Molinari NAM, Ortega-Sanchez IR, Messonnier ML, Thompson WW, Wortley PM, Weintraub E, et al. The annual impact of seasonal influenza in the US: measuring disease burden and costs. NPJ Vaccines. 2007 Jun 28;25(27):5086-96.

8. Executive Guideline of influenza and Influenza-like illness Surveillance in 2015 [Internet]. World Health Organization. 2016 [cited 24 September 2016]. Available from: baharestan.iums.ac.ir/uploads/ anfo_63891.pdf (in Persian)

9. Gouya M, Rezaei F, Haghdoost A, Nabavi M, Farahi KS, Mostafavi $\mathrm{E}$, et al. Estimation of influenza and severe acute respiratory illness incidence (burden) in three provinces of the Islamic Republic of Iran, 2012 and 2013. East Mediterr Health J. 2016;22(7):432.

10. Riazi H, Jafarpour M, Bitaraf E, editors. Towards National eHealth Implementation-A Comparative Study on WHO/ITU National eHealth Strategy Toolkit In Iran. MIE; 2014.

11. WHO surveillance case definitions for ILI and SARI [Internet]. World Health Organization. 2019 [cited 24 April 2019]. Available from: http://www.who.int/influenza/surveillance_monitoring/ili_sari_ surveillance case definition/en/

12. Flu Prevention: Information for Travelers [Internet]. Centers for Disease Control and Prevention. 2019 [cited 7 May 2019]. Available from: https://www.cdc.gov/flu/travelers/travelersfacts.htm

13. Cromley EK, McLafferty SL. GIS and public health: Guilford Press; 2011.

14. Jenks GF. Optimal data classification for choropleth maps. Department of Geographiy, University of Kansas Occasional Paper. 1977.
15. Anselin L. The local indicators of spatial association - LISA. Geogr Anal 1995;27:93-115.

16. Getis A, Ord JK. The analysis of spatial association by use of distance statistics. Perspectives on Spatial Data Analysis: Springer; 2010. p. 127-45.

17. Khoshabi M, Taleai M, Motlagh A, Kamal FH. Developing a WebGIS for Geo-Visualization of Cancer. Iran J Cancer Prev. 2016 Apr;9(2).

18. Jana M, Sar N. Modeling of hotspot detection using cluster outlier analysis and Getis-Ord Gi* statistic of educational development in upper-primary level, India. Model Earth Syst Environ. 2016;2(2):60.

19. Getis A, Ord JK. The analysis of spatial association by use of distance statistics. Perspectives on Spatial Data Analysis: Springer; 2010. p. 127-45.

20. Mitchell A. The ESRI Guide to GIS Analysis. Redlands (CA): ESRI Press; 2005.

21. Cromley RG, Cromley EK. Choropleth map legend design for visualizing community health disparities. Int $\mathrm{J}$ Health Geogr. 2009;8(1):52.

22. Całka B. Comparing continuity and compactness of choropleth map classes. Geodesy Cartogr. 2018;67. 